\title{
Ultra-Early Combination Antiplatelet Therapy with Cilostazol for the Prevention of Branch Atheromatous Disease: A Multicenter Prospective Study
}

\author{
Teruo Kimura $^{a}$ Adam Tucker $^{b}$ Toshihide Sugimura ${ }^{a}$ Toshitaka Seki ${ }^{a}$ \\ Shin Fukuda ${ }^{a}$ Satoru Takeuchi ${ }^{a}$ Shiro Miyata ${ }^{a}$ Tsutomu Fujita ${ }^{c}$ \\ Akira Hashizume $^{d}$ Naoto Izumi ${ }^{e}$ Kazutsune Kawasaki ${ }^{e}$ Makoto Katsuno ${ }^{e}$ \\ Masaaki Hashimoto $^{e}$ Kazuhiro Sako ${ }^{f}$ \\ ${ }^{a}$ Departments of Stroke Medicine and Neurosurgery, Dohtoh Neurosurgery Hospital and

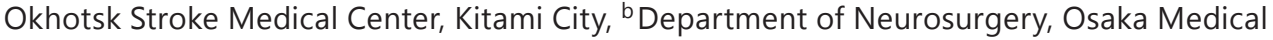 \\ College, Takatsuki, 'Departments of Stroke Medicine and Neurosurgery, Bihoro Clinic, Bihoro, \\ ${ }^{\mathrm{d}}$ Departments of Stroke Medicine and Neurosurgery, Katsuragaoka Clinic, and ${ }^{\mathrm{e}}$ Departments of \\ Stroke Medicine and Neurosurgery, Abashiri Neurosurgery Rehabilitation Hospital, Abashiri, and \\ ${ }^{f}$ Departments of Stroke Medicine and Neurosurgery, Nayoro Higashi Hospital, Nayoro, Japan
}

Key Words

Branch atheromatous disease · Cilostazol · Dual antiplatelet therapy · Progressive stroke

\begin{abstract}
Background and Purpose: The optimal use of antiplatelet therapy for intracranial branch atheromatous disease (BAD) is not known. Methods: We conducted a prospective multicenter, single-group trial of 144 consecutive patients diagnosed with probable BAD. All patients were treated within $12 \mathrm{~h}$ of symptom onset to prevent clinical progression using dual antiplatelet therapy with cilostazol plus one oral antiplatelet drug (aspirin or clopidogrel). Endpoints of progressive BAD in the dual therapy group at 2 weeks were compared with a matched historical control group of 142 patients treated with single oral antiplatelet therapy using either cilostazol, aspirin, or clopidogrel. Results: Progressive motor paresis occurred in 14 patients (9.7\%) in the aggressive antiplatelet group, compared with 48 (33.8\%) in the matched single antiplatelet group. Multivariate logistic regression analysis revealed the following variables to be associated with a better prognosis for BAD: baseline modified Rankin Scale score, dual oral antiplatelet therapy with cilostazol, and dyslipidemia (odds ratios of 0.616, 0.445, and 0.297, respectively). Hypertension was associated with a worse prognosis for BAD (odds ratio of 1.955). Conclusions: Our trial showed that clinical progression of BAD was significantly reduced with the administration of ultra-early aggressive combination therapy using cilostazol compared to treatment with antiplatelet monotherapy.



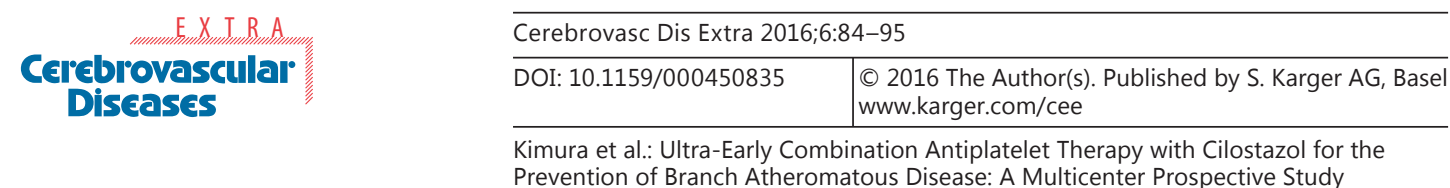

\section{Introduction}

Branch atheromatous disease (BAD) as previously described by Caplan [1], and recently characterized radiographically by the term branch occlusive disease by Ryoo et al. [2], is a well-known clinical phenomenon in Asia and especially in Japan, where reports range from $2.1 \%$ of lacunar and atherothrombotic infarctions to $13.1 \%$ of all cerebral infarctions [3-5]. The higher incidence may be explained by a greater prevalence of intracranial atherosclerosis and related small-vessel disease which has been ascribed to more sodium intake and uncontrolled hypertension than in Western populations [6]. Radiographically, early BAD can be confused with a lacunar infarction, and clinically BAD differs in that both symptoms and infarct size acutely progress. Likewise, the pathophysiological mechanisms are theoretically quite different from lacunar infarction; BAD is characterized by the presence of an atheromatous lesion in the parent artery at the orifice of penetrating branch arteries in the territories of the lenticulostriate arteries (LSA) and pontine paramedian arteries (PPA) where branch occlusion results in a progressively enlarging subcortical infarct measuring less than $15 \mathrm{~mm}$ (fig. 1a, b). Historically, pathological studies have been used to identify mechanisms of stroke; however, in recent years, high-resolution and high-field magnetic resonance imaging (MRI) have been used to further delineate the intracranial vessel wall morphology in small-vessel occlusive disease [7,8]. However, currently there is no corresponding cerebrovascular disorder classification in the National Institute of Neurological Disorders and Stroke (NINDS) [9] or diagnostic criteria in the Trial of Org 10172 in Acute Stroke Treatment (TOAST) [10]. Moreover, a recent review of BAD studies concluded that the fundamental definition of BAD as well as the mechanisms and rate of clinical progression are still unclear, yet $\mathrm{BAD}$ was frequently reported to be associated with early neurological deterioration [8].

BAD typically presents with an acutely progressive pure lacunar syndrome which is refractory to standard therapy once progression has begun $[11,12]$. Consequently, there is a great deal of frustration among both family and medical staff alike because the neurological status usually declines subsequent to admission, despite treatment. We have sought to investigate whether ultra-early treatment of BAD with a combination of cilostazol plus one oral antiplatelet drug (aspirin or clopidogrel) could prevent clinical progression of BAD.

\section{Subjects and Methods}

\section{Study Design}

In this prospective, multicenter, single-group cohort study, we compared ultra-early treatment of BAD initiated within $1 \mathrm{~h}$ of admission and within $12 \mathrm{~h}$ of onset using dual oral antiplatelet therapy [cilostazol plus one oral antiplatelet drug (aspirin or clopidogrel)] with a historical control group of BAD patients treated with single oral antiplatelet therapy (either cilostazol, aspirin, or clopidogrel). The study was conducted in Hokkaido, Japan, at the Dohtoh Neurosurgical Hospital, the Bihoro Clinic, the Katsuragaoka Clinic, the Abashiri Neurosurgical Rehabilitation Hospital, and the Nayoro Higashi Hospital. The study protocol was approved by the institutional review board and the ethics committee of the Kitami Medical Society (15B 128).

\section{Study Protocol}

Probable BAD was defined as a history of acute progressive hemiparesis prior to admission, and radiological evidence on admission of a high signal intensity measuring less than or equal to $15 \mathrm{~mm}$ in diameter on two or more consecutive axial MRI diffusion-weighted imaging (DWI) transverse slices in the white matter of the LSA territory (internal capsule 


\section{Cerebrovascular \\ Diseases}

\begin{tabular}{l|l}
\hline Cerebrovasc Dis Extra 2016;6:84-95 \\
\hline DOI: 10.1159/000450835 & $\begin{array}{l}\text { (c) 2016 The Author(s). Published by S. Karger AG, Basel } \\
\text { www.karger.com/cee }\end{array}$ \\
\hline
\end{tabular}

Kimura et al.: Ultra-Early Combination Antiplatelet Therapy with Cilostazol for the Prevention of Branch Atheromatous Disease: A Multicenter Prospective Study

and/or corona radiata), or in the PPA territory (ventral pons), with less than $50 \%$ of M1 stenosis on magnetic resonance angiography (MRA) and no evidence of atrial fibrillation. Patients were stratified according to infarction location (supratentorial or infratentorial), and finally enrolled in the study. Dual oral antiplatelet therapy was administered during the ultra-early phase within $1 \mathrm{~h}$ after arrival and within $12 \mathrm{~h}$ of onset. Eighty-nine patients received cilostazol (200 mg) and aspirin (300 mg, which was reduced to $100 \mathrm{mg}$ after day 3)

a

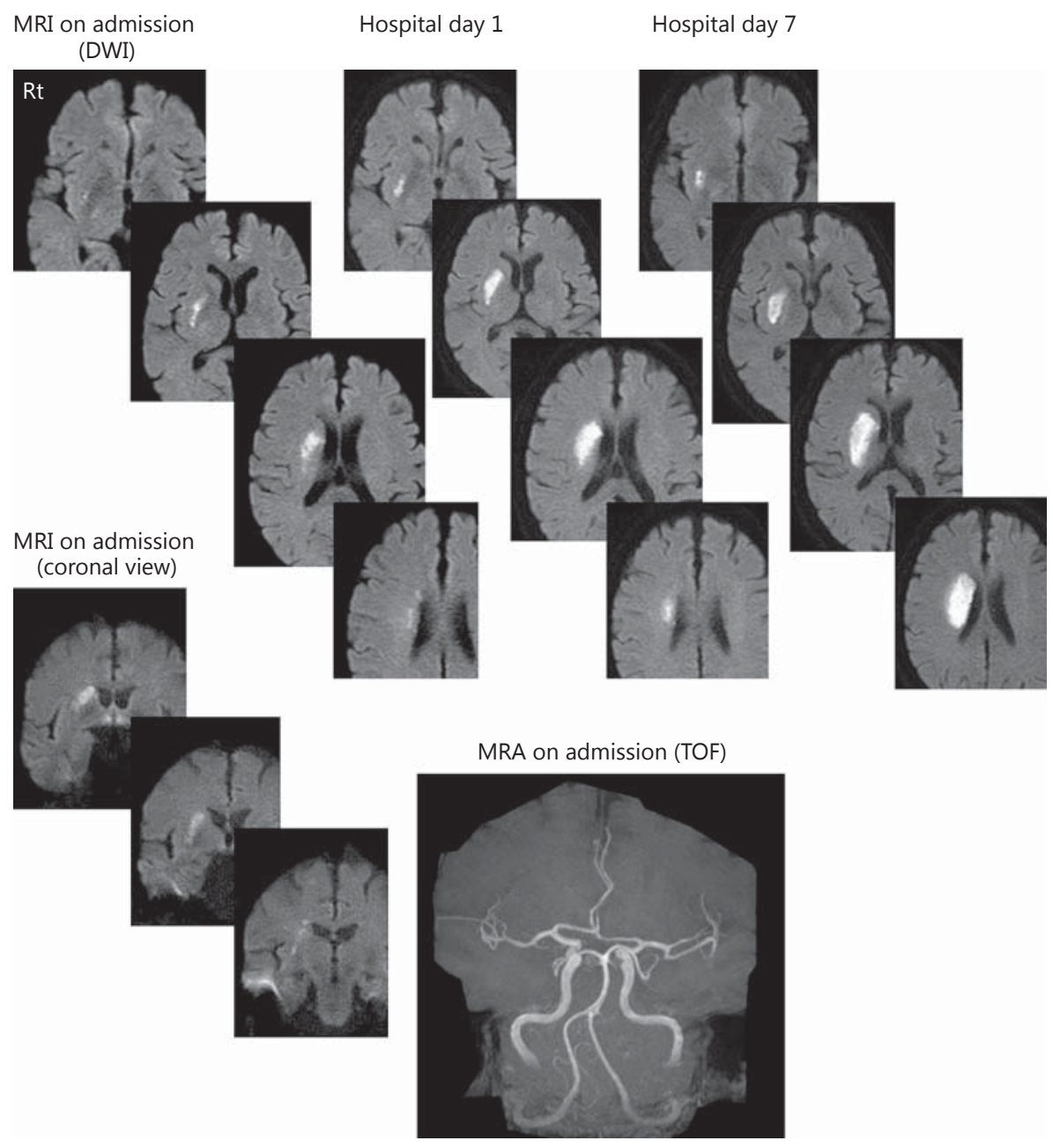

b

MRI on admission

(TOF)

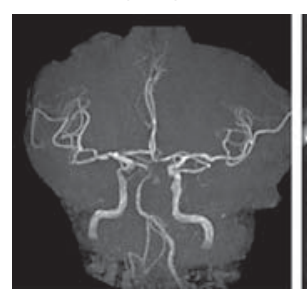

on admission

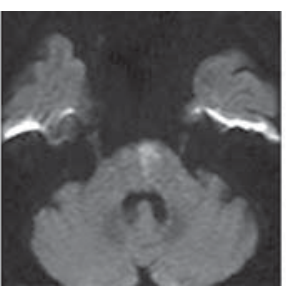

Hospital day 1

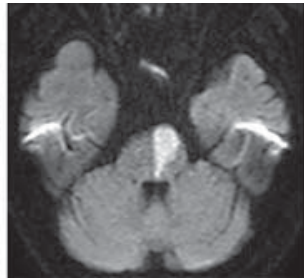

Hospital day 2

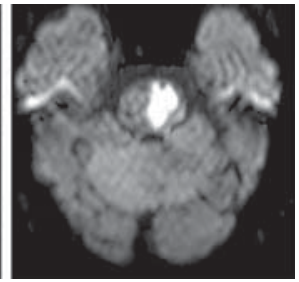

1

(For legend see next page.) 
and 65 patients received cilostazol $(200 \mathrm{mg}$ ) and clopidogrel (225 mg loading dose, which was reduced to $75 \mathrm{mg}$ after day 3) (fig. 2). Oral antiplatelet drugs were reduced to one drug after 7 days of combination therapy. A standard regimen of intravenous medication, including sodium ozagrel (160 mg/day) or argatroban (60 mg/day), edaravone (60 mg/day), and dextran $(500 \mathrm{ml} /$ day) was coadministered. Neurological examination and imaging were performed on admission, hospital day 1, day 7, and 2 weeks after admission (table 1). MRI studies included the following axial and coronal sequences: DWI, T2*-weighted imaging, T2-weighted imaging, fluid-attenuated inversion recovery and MRA.

\section{Selection of Historical Control Group}

A historical control group of adults receiving single oral antiplatelet medication was drawn from a total of 1,223 patients with a diagnosis of cerebral infarction. The patients received cilostazol (200 mg) or aspirin (300 mg, which was reduced to $100 \mathrm{mg}$ after day 3) or clopidogrel (225 mg loading dose, which was reduced to $75 \mathrm{mg}$ after day 3) (fig. 2). From these patients, 306 patients were diagnosed with lacunar infarct at the time of admission (supratentorial: 230, infratentorial: 76) and of these 142 were diagnosed with probable BAD (LSA region: 85, PPA region: 57) (table 1).

\section{Study Outcomes}

Primary endpoints included progression of BAD in the dual antiplatelet therapy group compared with progression of BAD in a matched historical control group of 142 patients treated with single oral antiplatelet therapy. Outcome in both groups was assessed using National Institutes of Health Stroke Scale (NIHSS) or modified Rankin Scale (mRS) scores, obtained by subtracting the values measured on hospitalization from those measured on hospital day 14. BAD with progressive motor paralysis (progressive BAD) was defined as an aggravation of 1 point or more in NIHSS or mRS admission scores compared with day 14 scores.

\section{Statistical Analysis}

Qualitative variables are expressed as absolute number and percentage. Results are expressed as mean \pm standard deviation (SD) for normally distributed data and as the median (interquartile range) of nonparametric data. Between-group comparisons for continuous variables were made using the unpaired t test and the Mann-Whitney U test. Categorical variables were made using Fisher's exact test. Logistic regression was employed to test whether the independent variables were predictive of prognosis. Variables showing a p value of less than 0.2 for univariate analysis were entered into a multivariate logistic analysis using the

Fig. 1. Single oral antiplatelet administration. a A 73-year-old man presented with left hand weakness during breakfast, and thereafter his hemiparesis progressively deteriorated. At noon, he was admitted to our hospital on foot but with apparent gait disturbance. Admission MRI DWI revealed a high signal intensity in the right LSA territory. MRA (time of flight, TOF) showed no stenosis of the right M1. A diagnosis of probable BAD was made, and monotherapy (clopidogrel $225 \mathrm{mg}$ ) and argatroban were started immediately. The next morning, his left hemiparesis progressed to hemiplegia and MRI (DWI) demonstrated an expanded lesion on hospital day 1, with further expansion on day 7. b An 81-year-old man was found to be unable to drive due to right extremity weakness and was taken immediately by his co-workers by car to our hospital. After admission, progression to mild right hemiparesis and dysarthria was observed. Admission MRI (DWI) showed a partial high intensity lesion in the left ventral pons and no basilar artery stenosis on MRA. A diagnosis of probable BAD was made and monotherapy (aspirin $300 \mathrm{mg}$ ) and argatroban were administrated immediately; however, he experienced progressive hemiplegia during the first night of admission and MRI (DWI) on hospital day 1 revealed lesion expansion, with further expansion on day 7 . 
Kimura et al.: Ultra-Early Combination Antiplatelet Therapy with Cilostazol for the Prevention of Branch Atheromatous Disease: A Multicenter Prospective Study

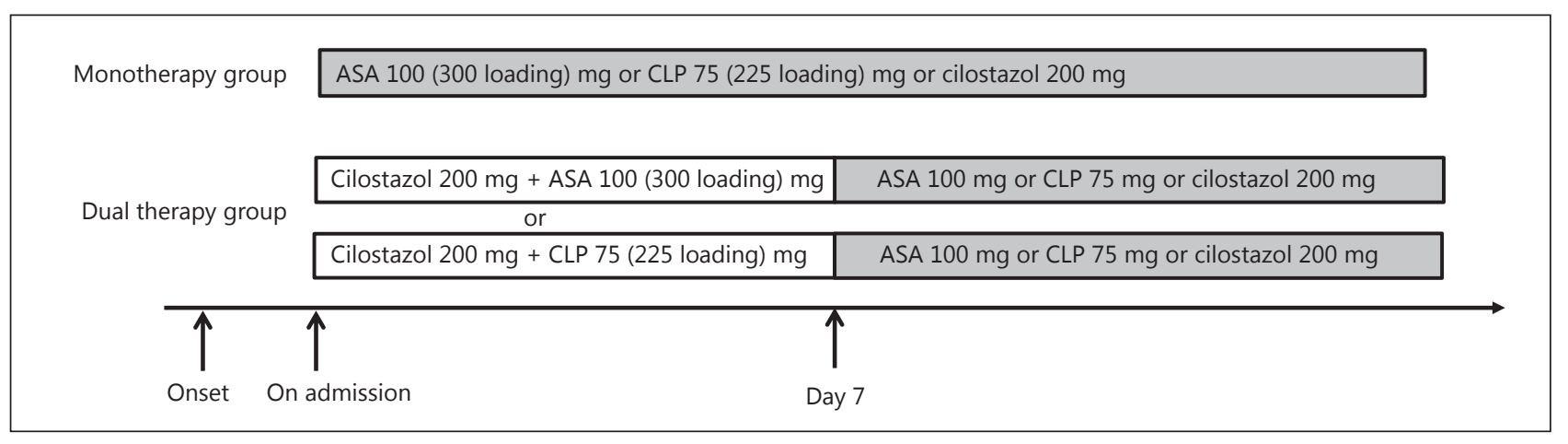

Fig. 2. Single oral antiplatelet administration. Patients in the monotherapy-matched group were treated with one oral antiplatelet drug plus an intravenous drug such as ozagrel, argatroban or edaravone. Therapy was initiated within $1 \mathrm{~h}$ from the time of hospital admission and within $12 \mathrm{~h}$ of onset. Patients in the aggressive dual antiplatelet group were given cilostazol (200 mg) plus aspirin (ASA, $300 \mathrm{mg}$ ) or cilostazol (200 mg) plus clopidogrel (CLP, $225 \mathrm{mg}$ loading reduced to $75 \mathrm{mg}$ after day 3 ) in addition to the standard intravenous protocol. Overall oral antiplatelet medication was decreased to one drug after 1 week.

forward selection method. A two-sided p value of less than 0.05 was considered statistically significant. Statistical analysis was performed with SPSS for Windows (version 22.0; IBM Japan, Tokyo, Japan).

\section{Results}

\section{Study Participants}

From a total of 959 cerebral infarction patients who participated in the trial between December 2011 to August 2014, 305 were initially diagnosed radiologically with lacunar infarction at the time of admission (supratentorial: 224, infratentorial: 81) and of these 144 showed acute clinical progression and were diagnosed with probable BAD and finally enrolled in the study. There were 83 men and 61 women with a median age of 72.0 years (range: 37-95). Radiological distribution included 102 patients with infarcts in the LSA area and 42 in the PPA area, with an average of 2.56 slices for probable BAD patients. Of those treated with dual antiplatelet therapy, 89 received cilostazol and aspirin, while 65 received cilostazol and clopidgrel. Six patients had already received clopidogrel prior to hospitalization for coronary stent implantation, and 4 had previously been taking clopidogrel for prevention of recurrent cerebral infarction. Dextran was used in 122 patients, ozagrel in 34, argatroban in 110, and edaravone in 131 patients, respectively. Statins were used in 43 patients, calcium antagonists in 53, and angiotensin II receptor blockers in 65. Patient background included the following: 85 patients had a history of hypertension (53 were receiving oral calcium antagonist medications and 65 were receiving angiotensin II receptor blockers), 40 had diabetes mellitus, 52 had dyslipidemia ( 43 were receiving oral statins), 10 had atrial fibrillation, 43 had a history of tobacco abuse, 11 were obese with a body mass index of 35 or higher, 10 had chronic kidney disease, and 4 had sleep apnea syndrome (table 1).

\section{Outcomes}

Nonprogressive Group

A total of 130 patients (men: 76, women: 54, age: 43-98 years, average age: 70.31 years) in the cilostazol-based dual therapy group who were diagnosed with probable BAD showed 
Table 1. Patient characteristics

\begin{tabular}{|c|c|c|c|}
\hline & $\begin{array}{l}\text { Matched monotherapy } \\
\text { antiplatelet group } \\
(\mathrm{n}=142)\end{array}$ & $\begin{array}{l}\text { Cilostazol-based dual } \\
\text { therapy group } \\
(n=144)\end{array}$ & $\mathrm{p}$ value \\
\hline Sex, n (\%) & & & $0.476^{\mathrm{b}}$ \\
\hline Male & $75(52.8)$ & $83(57.6)$ & \\
\hline Female & $67(47.2)$ & $61(42.4)$ & \\
\hline Median age [IQR] (mean), years & $73.0[61.8,80.0](71.0)$ & $72.0[62.0,79.0](72.0)$ & $0.627^{\mathrm{a}}$ \\
\hline Region, $\mathrm{n}(\%)$ & & & $0.062^{\mathrm{b}}$ \\
\hline LSA & $85(59.9)$ & $102(70.8)$ & \\
\hline PPA & $57(40.1)$ & $42(29.2)$ & \\
\hline Median baseline NIHSS score [IQR] (mean) & $3.0[2.0,4.0](3.1)$ & $3.0[3.0,4.0](3.4)$ & $0.043^{\mathrm{a}}$ \\
\hline Median baseline mRS score [IQR] (mean) & $2.0[1.0,2.0](1.9)$ & $3.0[2.0,3.0](2.9)$ & $0.000^{\mathrm{a}}$ \\
\hline \multicolumn{4}{|l|}{ Drug, n (\%) } \\
\hline Dextran & $136(95.8)$ & $122(84.7)$ & $0.002^{\mathrm{b}}$ \\
\hline Sodium ozagrel & $136(95.8)$ & $34(23.6)$ & $0.000^{\mathrm{b}}$ \\
\hline Argatroban & $8(5.6)$ & $110(76.4)$ & $0.000^{\mathrm{b}}$ \\
\hline Edaravone & $114(80.3)$ & $131(91.0)$ & $0.011^{\mathrm{b}}$ \\
\hline Cilostazol & $55(38.7)$ & $144(100.0)$ & $0.000^{\mathrm{b}}$ \\
\hline Clopidogrel & $77(54.2)$ & $65(45.1)$ & $0.156^{\mathrm{b}}$ \\
\hline Aspirin & $34,(23.9)$ & $89(61.8)$ & $0.000^{\mathrm{b}}$ \\
\hline Statin & $35(24.6)$ & $43(29.9)$ & $0.354^{\mathrm{b}}$ \\
\hline Angiotensin II receptor blocker & $66(46.5)$ & $65(45.1)$ & $0.906^{\mathrm{b}}$ \\
\hline Ca antagonist & $70(49.3)$ & $53(36.8)$ & $0.042^{\mathrm{b}}$ \\
\hline \multicolumn{4}{|l|}{ Previous history, n (\%) } \\
\hline Hypertension & $100(70.4)$ & $85(59.0)$ & $0.048^{\mathrm{b}}$ \\
\hline Diabetes mellitus & $44(31.0)$ & $40(27.8)$ & $0.604^{\mathrm{b}}$ \\
\hline Hyperlipidemia & $48(33.8)$ & $52(36.1)$ & $0.711^{\mathrm{b}}$ \\
\hline Atrial fibrillation & $4(2.8)$ & $10(6.9)$ & $0.169^{\mathrm{b}}$ \\
\hline Smoking & $29(20.4)$ & $43(29.9)$ & $0.077^{\mathrm{b}}$ \\
\hline Sleep apnea syndrome & $1(0.7)$ & $4(2.8)$ & $0.371^{\mathrm{b}}$ \\
\hline Obesity & $11(7.7)$ & $11(7.6)$ & $>0.999^{\mathrm{b}}$ \\
\hline Chronic kidney disease & $4(2.8)$ & $10(6.9)$ & $0.169^{\mathrm{b}}$ \\
\hline
\end{tabular}

LSA $=$ Lenticulostriate artery $\approx$ supratentorial $\mathrm{BAD} ; \mathrm{PPA}=$ pontine paramedian artery $\approx$ infratentorial BAD. ${ }^{\mathrm{a}}$ Mann-Whitney U test. ${ }^{\mathrm{b}}$ Fisher's exact test.

no evidence of clinical progression after admission (fig. 3). Of these, 94 had lesions in the LSA region and 36 in the PPA region. Overall NIHSS and mRS scores at the time of admission were 3.35 and 2.84, respectively, and improved on day 14 to 0.98 and 0.83 , respectively. NIHSS and $\mathrm{mRS}$ scores for patients with lesions in the LSA region at the time of admission were 3.34 and 2.84, respectively. These scores improved on day 14 to 0.91 and 0.81 , respectively. NIHSS and mRS scores for patients with lesions in the PPA region at the time of admission were 3.36 and 2.84, respectively, which improved on day 14 to 1.12 and 0.89 , respectively (table 2).

In the nonprogressive monotherapy-matched group, NIHSS and mRS scores for patients with lesions in the LSA region on admission were 2.84 and 1.81, respectively, which improved on day 14 to 2.30 and 1.46, respectively. NIHSS and mRS scores for patients with lesions in the PPA region on admission were 3.30 and 2.19, respectively, which improved on day 14 to 2.87 and 1.84 , respectively (table 2 ). 


\section{Cerebrovascular" \\ Diseases}

\begin{tabular}{l|l}
\hline Cerebrovasc Dis Extra 2016;6:84-95 \\
\hline DOI: 10.1159/000450835 & $\begin{array}{l}\text { (c) 2016 The Author(s). Published by S. Karger AG, Basel } \\
\text { www.karger.com/cee }\end{array}$ \\
\hline
\end{tabular}

Kimura et al.: Ultra-Early Combination Antiplatelet Therapy with Cilostazol for the Prevention of Branch Atheromatous Disease: A Multicenter Prospective Study

\section{Progressive Group}

In the dual therapy group, NIHSS and mRS scores for patients with lesions in the LSA region at the time of admission were 3.75 and 3.25, respectively, which worsened on day 14 to 6.0 and 4.5, respectively. NIHSS and mRS scores for patients with lesions in the PPA region at the time of admission were 3.5 and 3.5, respectively, which worsened on day 14 to 5.5 and 5.0 , respectively (table 2 ).

In the monotherapy-matched group with progressive motor paralysis (fig. 1a, b), overall NIHSS and mRS scores for patients at the time of admission with lesions in the LSA distribution were 3.28 and 1.79, respectively, and subsequent aggravation on day 14 revealed scores of 7.48 and 3.93, respectively. NIHSS and mRS scores for patients with lesions in the

\section{a}

MRI on admission (DWI)
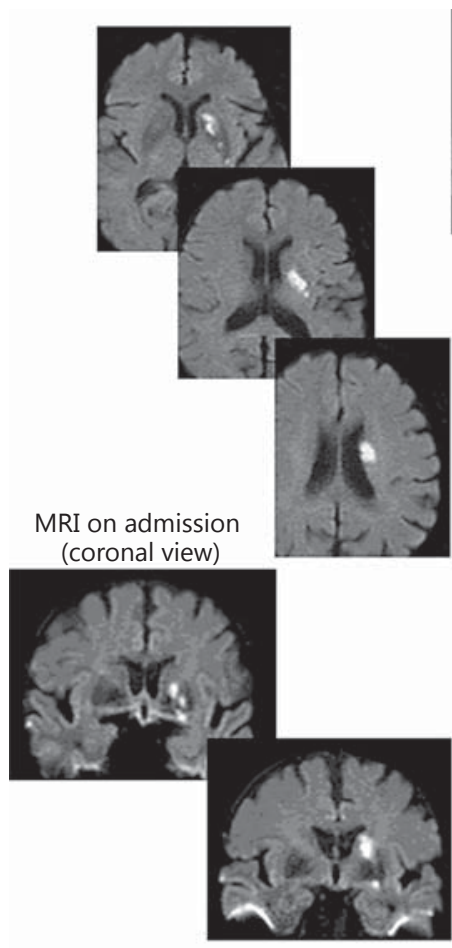

Hospital day 1

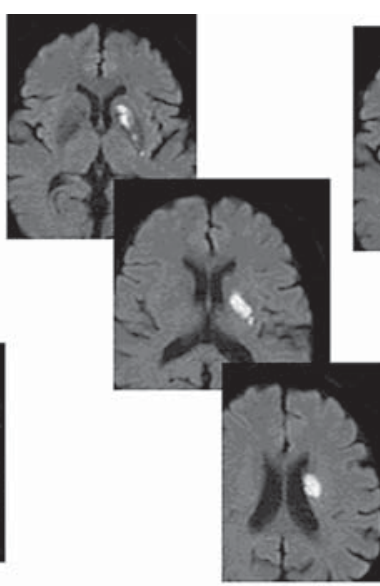

MRI on admission (TOF)
Hospital day 7

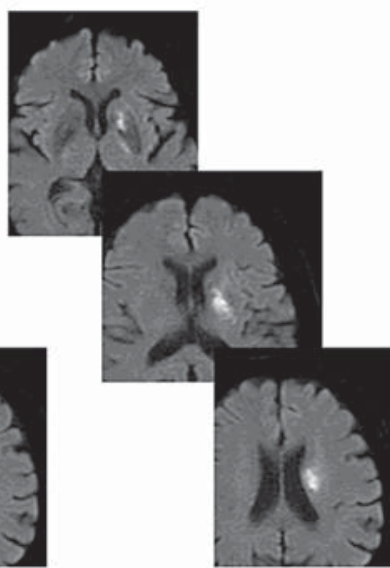

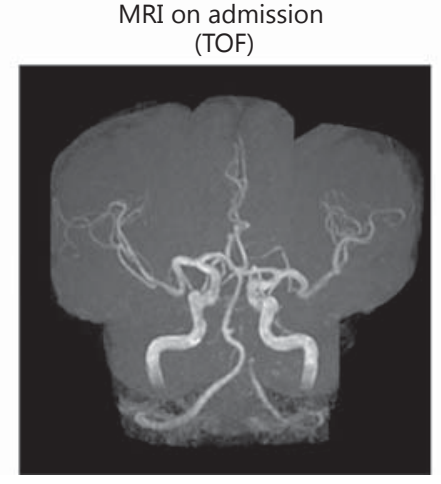

b

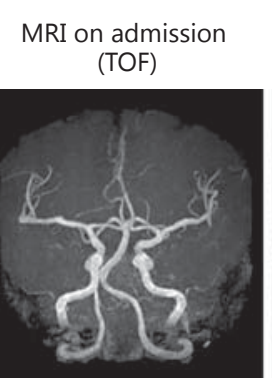

MRI on admission (DWI)

Hospital day 1

Hospital day 7
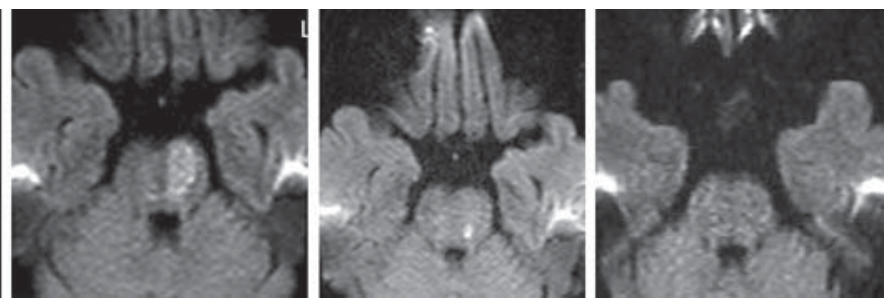

(For legend see next page.) 
PPA region were 3.00 and 1.94, respectively, and subsequent aggravation on day 14 revealed scores of 7.06 and 4.11, respectively (table 2).

Although $92.3 \%$ of all treatment combinations in the monotherapy-matched group were ineffective for stopping progression when initiated after the onset of progression, in 48 patients with progressive cerebral infarction, progression was inhibited to some extent in only 3 patients, all of whom had received oral cilostazol. With the use of only one antiplatelet drug in addition to an intravenous medication, 30 out of 85 patients with BAD in the LSA region (35.3\%) and 18 out of 57 patients with BAD in the PPA region (31.6\%) suffered progressive stroke, despite medication initiation prior to progression onset.

\section{Comparison of Outcomes}

Forty-nine of the 142 patients in the monotherapy-matched group (33.8\%) showed progression of BAD after initiation of therapy, while only 14 out of 144 patients $(9.7 \%)$ in the

Table 2. Comparison of outcomes: cilostazol-based dual therapy group $(n=144)$ versus matched monotherapy antiplatelet group $(\mathrm{n}=142)$

\begin{tabular}{|c|c|c|c|c|c|c|}
\hline & $\begin{array}{l}\text { Admission } \\
\text { (total) }\end{array}$ & $\begin{array}{l}\text { Day } 14 \\
\text { (total) }\end{array}$ & $\begin{array}{l}\text { LSA } \\
\text { admission }\end{array}$ & $\begin{array}{l}\text { LSA } \\
\text { day } 14\end{array}$ & $\begin{array}{l}\text { PPA } \\
\text { admission }\end{array}$ & $\begin{array}{l}\text { PPA } \\
\text { day } 14\end{array}$ \\
\hline \multicolumn{7}{|c|}{ Progressive $B A D$} \\
\hline \multicolumn{7}{|c|}{ Aggressive group ( $\mathrm{n}=14$ : LSA 8, PPA 6) } \\
\hline NIHSS & 3.64 & 5.79 & 3.75 & 6 & 3.5 & 5.5 \\
\hline $\mathrm{mRS}$ & 3.36 & 4.71 & 3.25 & 4.5 & 3.5 & 5 \\
\hline \multicolumn{7}{|c|}{ Monotherapy group $(n=49:$ LSA 31, PPA 18) } \\
\hline NIHSS & 3.18 & 7.33 & 3.28 & 7.48 & 3 & 7.06 \\
\hline mRS & 1.85 & 4 & 1.79 & 3.93 & 1.94 & 4.11 \\
\hline \multicolumn{7}{|c|}{ Nonprogressive $B A D$} \\
\hline \multicolumn{7}{|c|}{ Aggressive group ( $\mathrm{n}=130$ : LSA 94, PPA 36) } \\
\hline NIHSS & 3.35 & 0.98 & 3.34 & 0.91 & 3.36 & 1.12 \\
\hline $\mathrm{mRS}$ & 2.84 & 0.83 & 2.84 & 0.81 & 2.84 & 0.89 \\
\hline \multicolumn{7}{|c|}{ Monotherapy group $(n=93:$ LSA 52, PPA 41) } \\
\hline NIHSS & 3.04 & 2.54 & 2.84 & 2.3 & 3.3 & 2.87 \\
\hline $\mathrm{mRS}$ & 1.98 & 1.62 & 1.81 & 1.46 & 2.19 & 1.84 \\
\hline
\end{tabular}

Fig. 3. Aggressive dual oral antiplatelet administration. a A 72-year-old women complained of speech difficulty with right hand weakness after lunch, and was admitted to our hospital in the evening on foot with unsteady gait. The neurological examination on admission revealed dysarthria and mild right hemiparesis. MRI (DWI) on admission showed a high intensity in the left LSA territory, with no stenosis of the M1 on MRA (time of flight, TOF). A diagnosis of probable BAD in the left LSA territory was made. Dual antiplatelet medication (cilostazol $200 \mathrm{mg}$ and clopidogrel $225 \mathrm{mg}$ ) and argatroban was administrated immediately. On the next day, the dysarthria and right hemiparesis improved, and there was no evidence of lesion expansion on hospital day 1 or day 7 MRI (DWI). She was discharged home on hospital day 14 with no deficits. b A 76-year-old woman complained of speech difficulty and gait disturbance, and was immediately admitted on foot to our hospital. On admission, her neurological examination showed mild dysarthria, mild right hemiparesis, and mild hemidysethesia. MRI (DWI) on admission showed a pale high-intensity lesion throughout the left PPA territory in the pons without basilar artery stenosis on MRA (TOF). A diagnosis of probable BAD in the left PPA territory of the pons was made and dual antiplatelet medication (cilostazol $200 \mathrm{mg}$ and clopidogrel 225 $\mathrm{mg}$ ) with argatroban was administrated immediately. On hospital day 1, her symptoms improved, and MRI (DWI) showed a spotty high-intensity lesion in the ventral pons. She was discharged home on hospital day 14 with only mild hemidysesthesia. 
dual antiplatelet group showed progression. Statistically significant patient differences between the monotherapy group and the dual therapy groups included the following: NIHSS and mRS scores at baseline; intravenous dextran, ozagrel, argatroban, and edaravone; oral cilostazol, aspirin, calcium antagonists, and a history of hypertension and obesity (table 1).

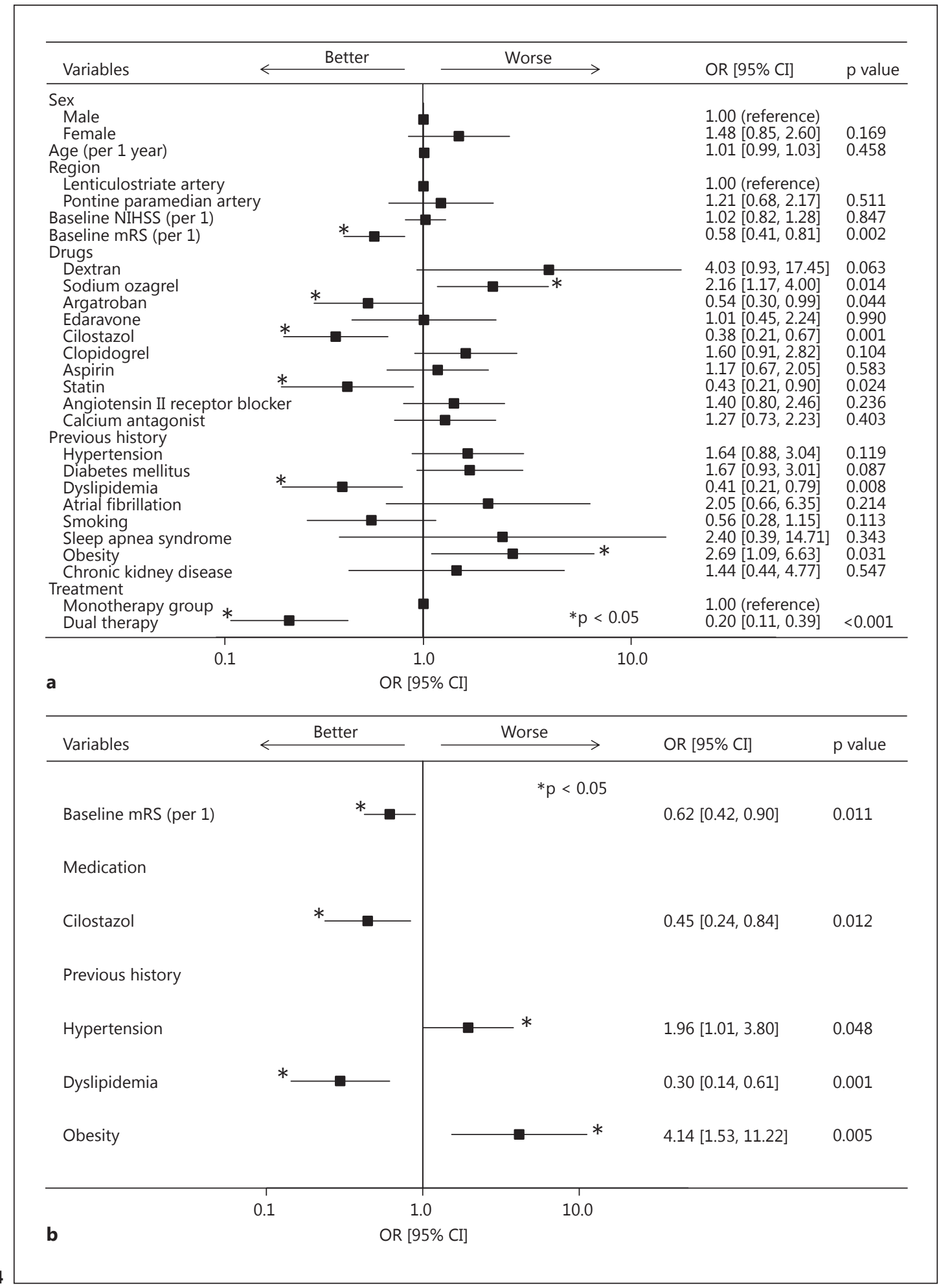


Univariate logistic regression analysis revealed that mRS at baseline, ozagrel and argatroban as injection drugs, cilostazol and statins as oral drugs, and a prior history of hypertension and obesity were statistically significant prognostic factors for BAD. The odds ratio for increased mRS at baseline was 0.580 (95\% CI 0.414-0.813). The odds ratio for ozagrel relative to no ozagrel was 2.160 (95\% CI 1.167-3.999), for argatroban relative to no argatroban it was 0.541 (95\% CI 0.297-0.985), for cilostazol relative to no cilostazol it was 0.378 (95\% CI 0.212-0.674), for statins relative to no statins it was 0.430 (95\% CI 0.207-0.896), for dyslipidemia relative to no dyslipidemia it was 0.406 (95\% CI 0.209-0.792), and for obesity relative to no obesity it was 2.692 (95\% CI 1.094-6.628), respectively (fig. 4a). Multivariate logistic regression analysis revealed mRS at baseline, oral cilostazol, and dyslipidemia as significant factors associated with a better prognosis of $\mathrm{BAD}$, while hypertension and obesity were significant factors associated with worse BAD prognosis. A $\chi^{2}$ test revealed the multivariate model coefficient to be significant at a level of $\mathrm{p}<0.001$. The odds ratio (95\% CI) for increased mRS scores at baseline was 0.616 (95\% CI $0.423-0.897$ ), for cilostazol relative to no use of cilostazol it was 0.445 (95\% CI 0.237-0.837), and for dyslipidemia relative to no history of dyslipidemia it was 0.297 (95\% CI 0.144-0.614), respectively (fig. 4b). The odds ratio (95\% CI) for hypertension relative to no history of hypertension was 1.955 (95\% CI 1.006-3.800), for dyslipidemia relative to no history of dyslipidemia it was 0.297 (95\% CI $0.144-0.614)$, and for obesity relative to no presence of obesity it was 4.143 (95\% CI 1.530-11.215), respectively (fig. 4b).

\section{Adverse Events}

Cerebral microbleeds were observed in a total of 39 patients in the dual therapy group with 5 or more microbleeds in 8 of the 39 patients. In 1 patient with 7 cerebral microbleeds, a small asymptomatic cerebral bleed was noted on MRI in the nucleus basalis on day 7. No symptomatic intracranial or systemic hemorrhagic complications were found.

\section{Discussion}

Although BAD is typically progressive and intractable to standard treatment protocols [13], studies have shown significant increases in coagulation and fibrinolysis activation as well as platelet function in patients with progressive cerebral infarction or those with large lacunar infarct areas, suggesting a possible role for combination antiplatelet and anticoagulant drugs [14]. Caplan [15] has recommended treatment using antiplatelet drugs, especially the use of cilostazol for penetrating artery disease, as well as other rheological measures, based on his anatomical and pathophysiological observations of penetrating and branch artery disease. A small study of $60 \mathrm{BAD}$ patients using combination therapy with argatroban, edaravone and cilostazol compared monotherapy in 120 BAD patients and found a significant improvement in mRS scores at discharge in the combination therapy group [16].

Fig. 4. a Univariate logistic regression for prognosis of BAD. Univariate logistic regression analysis revealed the following statistically significant prognostic factors for BAD: mRS at baseline; ozagrel and argatroban as intravenous drugs; oral cilostazol and statin medication, and a previous history of hypertension and obesity. b Multivariate logistic regression for the prognosis of BAD. Multivariate logistic regression analysis revealed the following statistically significant prognostic factors for BAD: mRS at baseline; oral cilostazol; a previous history of hypertension, dyslipidemia, and obesity. $\chi^{2}$ test for multivariate model coefficient: $\mathrm{p}<0.001$. Selection of variables: forward selection method using likelihood ratio. Variables used: baseline mRS, dextran, ozagrel $\mathrm{Na}$, cilostazol, clopidogrel, statin, hypertension, diabetes mellitus, dyslipidemia, smoking, and obesity. Variables seen in univariate analysis: $\mathrm{p}<0.2$. 

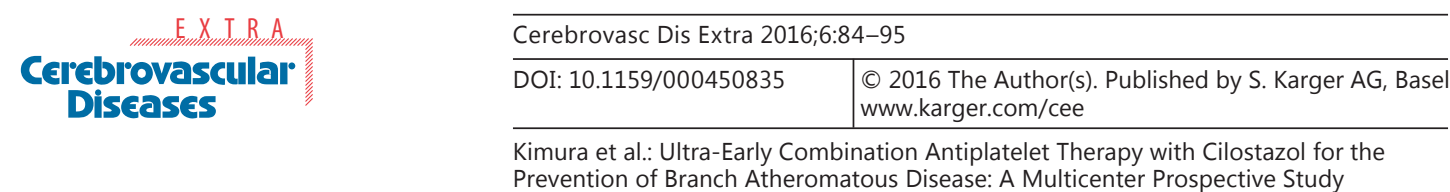

In our trial, we evaluated the ultra-early use of dual antiplatelet therapy for halting progression of BAD in anatomical areas associated with a high likelihood of progressive motor paralysis. Previous studies have shown evidence of clinical progression predominantly in the LSA and PPA regions. Yamada et al. [17] reported that 16 out of 61 patients with penetrating branch infarction showed progression, 14 of whom had an infarct area measuring 15 $\mathrm{mm}$ or larger, corresponding to the definition of BAD in our study. In addition, according to the results from the J-BAD Registry, a Japanese multicenter study, patients showing aggravation of neurological symptoms by 1 point or more in the NIHSS accounted for $30.1 \%$ of patients with infarctions in the LSA distribution and $38.3 \%$ of patients in the PPA distribution [18]. Umemura et al. [5] reported that progression of BAD was observed in 23.0 and $41.7 \%$ of supratentorial and infratentorial lesions, respectively. Tanaka et al. [13] reported 12 out of 15 patients with pontine BAD, and Hoshino et al. [4] reported that $30.1 \%$ of BAD in the LSA region and $43.6 \%$ of $\mathrm{BAD}$ in the PPA region led to cerebral stroke even after initiation of treatment.

In our study, the following factors were statistically significantly associated with better prognosis in BAD patients: dual antiplatelet therapy with cilostazol, superiority of argatroban over ozagrel, a previous history of dyslipidemia and oral statin treatment. In contrast, we found that a high mRS score on admission was predictive of disease progression and showed less likelihood of recovery. The use of statins for the prevention of atherosclerosis as well as for inhibition of BAD progression has been indicated in prior reports [19, 20]. Our results showed that hypertension was associated with worse prognosis for BAD, and although no reports are available to date on the relationship between hypertension and the prognosis of $\mathrm{BAD}$, hypertension has been reported to be a contributory factor for progressive cerebral infarction [21]. Regarding obesity, although a significant difference was shown in multivariate analysis, perhaps due to the smaller sample size compared to other factors, obesity may be less likely to be a direct factor involved in the poor BAD prognosis. Moreover, it is interesting to note that results from a recent review by Inzitari and colleagues [8] showed no consistent associated vascular risk factors or predictors of progression for BAD. Finally, reports on the use of intravenous medication have been controversial. A significant difference was observed between ozagrel and argatroban, although Fukuuchi et al. [22] indicated in a comparative clinical study that argatroban has a similar clinical effect to ozagrel in the acute stage of cerebral thrombosis.

Based on the above findings, ultra-early use of dual oral antiplatelet therapy, especially combinations with cilostazol, in addition to the use of statins and intravenous argatroban, would be effective for preventing BAD progression. However, long-term dual antiplatetelet therapy (aspirin and clopidogrel) for ischemic stroke may increase hemorrhagic complications [23]. Currently, there is no clinical data on hemorrhagic events associated with a combination use of cilostazol and other antiplatelet drugs in the acute phase.

\section{Limitations}

Our study has several methodological limitations. Data were collected via a prospective clinical register of consecutive patients; therefore, there are the inherent drawbacks of observational design. Secondly, the patient series is based on a related group center cohort, and even though the baseline profile is comparable to that of other series, we cannot exclude the possibility of systematic referral bias interfering with patient characteristics and clinical event profiles. Another important limitation is the lack of randomization. After completion of the historical control arm, a randomized design was attempted in 8 patients; however, superiority in the intervention group was demonstrated and the ethics committee supported a subsequent prospective design. Finally, imaging data was read by neurosurgeons, neurologists, and radiologists at the treating centers, with no independent verification. 


\begin{tabular}{l|l}
\hline Cerebrovasc Dis Extra 2016;6:84-95 \\
\hline DOI: $10.1159 / 000450835$ & $\begin{array}{l}\text { C) 2016 The Author(s). Published by S. Karger AG, Basel } \\
\text { www.karger.com/cee }\end{array}$ \\
\hline
\end{tabular}

Kimura et al.: Ultra-Early Combination Antiplatelet Therapy with Cilostazol for the Prevention of Branch Atheromatous Disease: A Multicenter Prospective Study

\section{Conclusions}

Aggressive combination antiplatelet therapy, using cilostazol with aspirin or clopidogrel, initiated during the ultra-early phase in patients with BAD was more effective for preventing progression of motor paralysis by almost one quarter compared to single antiplatelet therapy.

\section{Disclosure Statement}

The authors declare that there are no conflicts of interest.

\section{References}

1 Caplan LR: Intracranial branch atheromatous disease: a neglected, understudied, and underused concept. Neurology 1989;39:1246-1250.

-2 Ryoo S, Lee MJ, Cha J, Bang OY: Differential vascular pathophysiologic types of intracranial atherosclerotic stroke: a high-resolution wall magnetic resonance imaging study. Stroke 2015;46:2815-2821.

3 Adachi T, Kobayashi S: Data 2015 Stroke Bank (in Japanese). Tokyo, Nakayama Shoten, 2015, pp 116.

4 Hoshino H, Takagi M, Yamamoto Y, Ishibashi Y, Terayama Y, Takeda H, et al: Neurological progression and clinical outcome of branch atheromatous disease (in Japanese). Jpn J Stroke 2011;33:37-44.

5 Umemura T, Matsui K, Niimi Y, Umemura K, Terao S, Hirayama M: Clinical analysis of related factors for progression and prognosis in branch atheromatous disease (BAD) (in Japanese). Jpn J Stroke 2008;30:462-470.

6 Kim BJ, Kim JS: Ischemic stroke subtype classification: an Asian viewpoint. J Stroke 2014;16:8-17.

7 Chung JW, Kim BJ, Sohn CH, Yoon BW, Lee SH: Branch atheromatous plaque: a major cause of lacunar infarction (high-resolution MRI study). Cerebrovasc Dis Extra 2012;2:36-44.

8 Petrone L, Nannoni S, Del Bene A, Palumbo V, Inzitari D: Branch atheromatous disease: a clinically meaningful, yet unproven concept. Cerebrovasc Dis 2016;41:87-95.

-9 Special report from the National Institute of Neurological Disorders and Stroke. Classification of cerebrovascular diseases III. Stroke 1990;21:637-676.

$>10$ Adams HP Jr, Bendixen BH, Kappelle LJ, Biller J, Love BB, Gordon DL, et al: Classification of subtype of acute ischemic stroke. Definitions for use in a multicenter clinical trial. TOAST. Trial of Org 10172 in Acute Stroke Treatment. Stroke 1993;24:35-41.

11 Steinke W, Ley SC: Lacunar stroke is the major cause of progressive motor deficits. Stroke 2002;33:15101516.

12 Takagi M: Branch atheromatous disease (in Japanese). Neurol Med 2008;69:542-549.

-13 Tanaka H, Saito J, Isoe K, Watanabe Y, Fukada M: Clinical characteristics of pontine infarction due to occlusion of the mouth of the paramedian branches (in Japanese). Jpn J Stroke 1995;17:51-57.

14 Yamamoto Y, Oiwa K, Hayashi M, Imai K, Nakamura T: Coagulation and fibrinolytic activation in lacunar infarct patients (in Japanese). Clin Neurol 1999;39:1104-1108.

15 Caplan LR: Penetrating and branch artery disease; in Caplan LR: Caplan's Stroke: A Clinical Approach, ed 4. Philadelphia, Saunders Elsevier Health Sciences, 2009, pp 291-315.

16 Yamamoto Y: Topic of branch atheromatous disease (in Japanese). Pharma Medica 2008;26:160-165.

-17 Yamada K, Kato B, Takaishi S, Hirayama T, Hasegawa Y: Early neurological progression of acute small vessel infarction (in Japanese). Jpn J Stroke 2008;30:50-54.

-18 Hoshino H, Takagi M, Yamamoto Y, Ishibashi Y, Terayama Y, Takeda H, et al: Neurological progression and clinical outcome of branch atheromatous disease (result from the J-BAD registry) (in Japanese). Clin Neurol 2010;50:919-920.

19 Amareco P, Labreuche J: Lipid management in the prevention of stroke: review and updated meta-analysis of statins for stroke prevention. Lancet Neurol 2009;8:453-463.

20 Brugts JJ, Yetgin T, Hoeks SE, Gotto AM, Shepherd J, Westendorp RG, et al: The benefits of statins in people without established cardiovascular disease but with cardiovascular risk factors: meta-analysis of randomized controlled trials. BMJ 2009;339:26-29.

-21 Castillo J, Leira R: Predictors of deteriorating cerebral infarct: role of inflammatory mechanisms. Would its early treatment be useful? Cerebrovasc Dis 2001;11(suppl 1):40-48.

22 Fukuuchi Y, Tohgi H, Shinohara Y, Kobayashi S, Fujishima Y, Tazaki N, et al: A controlled clinical trial to assess the efficacy and safety of argatroban in treating acute cerebral thrombosis in comparison to sodium ozagrel (in Japanese). Neurol Ther 2001;18:273-282.

23 Diener HC, Bogousslavsky J, Brass LM, Cimminiello C, Csiba L, Kaste M, et al: Aspirin and clopidogrel compared with clopidogrel alone after recent ischemic stroke or transient ischemic attack in high-risk patients (MATCH): randomized, double-blind, placebo-controlled trial. Lancet 2004;364:331-337. 